\title{
The number-weight illusion
}

\author{
Wolf Schwarz ${ }^{1}$. Dennis Reike ${ }^{1}$
}

Published online: 11 May 2018

(C) Psychonomic Society, Inc. 2018

\begin{abstract}
When objects are manually lifted to compare their weight, then smaller objects are judged to be heavier than larger objects of the same physical weights: the classical size-weight illusion (Gregory, 2004). It is also well established that increasing numerical magnitude is strongly associated with increasing physical size: the number-size congruency effect e.g., (Besner \& Coltheart Neuropsychologia, 17, 467-472 1979; Henik \& Tzelgov Memory \& Cognition, 10, 389-395 1982). The present study investigates the question suggested by combining these two classical effects: if smaller numbers are associated with smaller size, and objects of smaller size appear heavier, then are numbered objects (balls) of equal weight and size also judged as heavier when they carry smaller numbers? We present two experiments testing this hypothesis for weight comparisons of numbered (1 to 9) balls of equal size and weight, and report results which largely conform to an interpretation in terms of a new "number-weight illusion".
\end{abstract}

Keywords Size-weight illusion - Number-size congruency effect · Numerical distance effect · Paired comparison · Reafference principle

The classical size-weight illusion is one of the most striking and robust demonstrations in perception: when objects (e.g., balls) are lifted manually, a smaller object feels and is judged to be heavier than a larger object of the same physical weight (for background and review, see Flanagan \& Beltzner, 2000; Gregory, 2004, pp. 842f; Walker, Francis, \& Walker, 2010). The size-weight illusion is often interpreted as a significant example of the general reafference principle (Gregory, 2015, ch. 6; von Helmholtz, 1910, ch. 26; von Holst \& Mittelstaedt, 1950), which postulates a comparison process of the internal ex-afferent signal (the efference copy) and the received

Electronic supplementary material The online version of this article (https://doi.org/10.3758/s13423-018-1484-z) contains supplementary material, which is available to authorized users.

Wolf Schwarz

wschwarz@uni-potsdam.de

Dennis Reike

reike@uni-potsdam.de

1 Department of Psychology, University of Potsdam, PO Box 60 15 53, 14415, Potsdam, Germany neural signals (the sensory re-afference). That is, a bigger ball is expected to be heavier, grip and load forces of the motor system are scaled accordingly, and if the sensory signals received do not match the internal expectation then the smaller ball is judged to be heavier. A striking example of how these ex-afferent expectations serve to adjust and calibrate our motor system is when we forcefully pick up an empty suitcase assumed to be full, which then flies in the air. More recent research (e.g., Flanagan \& Beltzner, 2000) demonstrated that the illusion persists even after people have learned to scale their fingertip forces precisely for the true object weights, which suggests that the reafference account does not necessarily (or exclusively) apply to the sensorimotor level but operates as well at a cognitive or perceptual level (for a detailed recent review of accounts of the size-weight illusion, see Dijker, 2014). Many studies investigating perceived weight focus on the influence of physical (e.g., Buckingham, Goodale, White, \& Westwood, 2016; Walker, Francis, \& Walker, 2010) or task-related (e.g., Masin \& Crestoni, 1988) variables on the size of the illusion. Surprisingly few studies have asked if the illusion also operates when the sizes of the objects differ only apparently, and if differences in apparent weight can be induced by purely symbolic, learned associations via differences in apparent size. The present study addresses these issues for a 
specific form of learned associations: those based on digits which symbolize numerical magnitudes.

Numbers and quantitative information have crucial significance in many contexts, and humans process with extreme efficiency numerical magnitude information as conveyed in abstract formats, such as digits. Correspondingly, the way in which we learn, retrieve, process, and compare numerical magnitudes as symbolized by digits is among the best-investigated processes in cognitive psychology. According to a prominent model of number processing (e.g., Dehaene \& Brannon, 2011; Maloney et al., 2010; Nieder, 2005; Reike \& Schwarz, 2016; Rugani \& de Hevia, 2017; Walsh, 2003), humans automatically convert digits into analog representations much like sensory representations of physical attributes such as brightness or size. These representations are often thought to be preverbal and highly over-learned, and to have a quasi-spatial format, a "mental number line" that preserves ordinal relations. On this mental number line, smaller numbers are separated from their neighbors (e.g., 2-3) more distinctly than larger numbers (e.g., 7-8). This latter effect is reminiscent of Weber's law in judging physical attributes, and is typically modeled by assuming that the numbers 1 to 9 are internally represented on a mental number line that is logarithmically compressed (Dehaene, 2003; Gallistel \& Gelman, 2005; Galton, 1880; Nieder, 2005; Reike \& Schwarz, 2016). Convergent evidence supporting this view comes from experimental, comparative, physiological, and developmental psychology and has been extensively reviewed (Butterworth, 1999; Dechaene \& Brannon, 2011; Whalen, Gallistel, \& Gelman, 1999).

A significant line of evidence supporting the assumption of an analog representation of number is the finding that small/large numerical magnitudes are consistently associated with small/large physical sizes, respectively. For example, humans are faster to choose the larger number in the pair $2-8$, as compared to $2-8$ (Besner \& Coltheart, 1979); the same association of number and size is also revealed in the inverse task to choose the physically larger digit (e.g., Cohen Kadosh et al., 2007; Henik \& Tzelgov, 1982; Reike \& Schwarz, 2017; Schwarz \& Ischebeck, 2003). Consistent associations with numerical magnitude have been documented for several other stimulus (e.g., time, Bonato, Zorzi, \& Umilta, 2012; Gevers, Reynvoet, \& Fias, 2003; Müller \& Schwarz, 2008; Schwarz \& Eiselt, 2009) and response dimensions (Fischer \& Miller, 2008); Lindemann et al., 2007; Rugani et al., 2017). These findings have been synthesized by Walsh (2003) in the general ATOM (a theory of magnitude) model according to which processing mechanisms related to quantity, space, and time all form part of a generalized magnitude system (also see Dehaene \& Brannon, 2011; Fitousi, 2014; Gallistel \& Gelman, 2000; Henik, Leibovich, Naparstek, Diesendruck, \& Rubinsten, 2012). More recent refined elaborations and computational formulations of the ATOM model have been presented, e.g., by Bueti and Walsh (2009), and by Lambrechts et al. (2013). Of specific interest in the present context is the finding of Andres et al. (2008) who demonstrated that digit magnitude processing shares common mechanisms with object size estimation during grasping. Specifically, under conditions quite similar to the present study, these authors showed that when humans grasp objects their grip aperture is larger consequent to the presentation of digits with a high value rather than a low one (for related results, see Lindemann et al., 2007). Similarly, Viarouge and de Hevia 2013 showed that when humans reproduce the physical size of a square, taskirrelevant larger numbers (8/9) presented at the corners of the square elicit larger size estimates in a size reproduction task than smaller numbers $(1 / 2)$ whereas no such effect is found for task-irrelevant letters (A/B vs. Y/Z). These studies represent strong, direct evidence supporting the view that the perception of the size of physical objects is congruently related to the numerical magnitude of digits attached to these objects.

The present study. The major aim of the present study is to combine the two separate strands of research reviewed above, following a simple syllogism. If smaller numerical magnitude is consistently associated with smaller physical size, and if smaller objects are consistently judged to be heavier than larger objects of the same weight, then we hypothesize that objects carrying smaller numbers will also be consistently judged as heavier than otherwise identical objects carrying larger numbers. Note that this relation could be seen as a priori implausible, as it is contrary to the standard finding that larger numerical magnitude goes with more of a perceived physical quantity. We will call this hypothesized relation for short the "number-weight illusion", and tested it in two complete paired-comparison experiments. In each experiment the comparison objects were balls numbered from 1 to 9 , and the task was to judge which one is heavier (Experiment 1) or lighter (Experiment 2). Unbeknownst to the participants all balls were of identical size and weight.

\section{Experiment 1}

Experiment 1 was a complete paired-comparison task in which participants judged which of two numbered balls appeared to be heavier. 


\section{Method}

\section{Participants}

Thirty-six University of Potsdam students, aged 18 to 33, participated individually in one session lasting approximately $30 \mathrm{~min}$. They either received a payment of $€ 8$ or course credit for their participation.

\section{Stimuli and apparatus}

The stimuli were nine red snooker balls (Aramith Premier; diameter $52.4 \mathrm{~mm}$, weight $129 \mathrm{~g}$ ), to which commercial adhesive numbers (height of $3 \mathrm{~cm}$ ) from 1 to 9 were permanently attached.

\section{Procedure}

After entering the experimental room, the Experimenter (using a standardized instruction script) explained the weight comparison task to the participants, and presented to them several balls carrying single-digit numbers on them as representative stimulus examples. In particular, they were shown several balls carrying the same number so as to create the clear impression that for every number there existed several distinct balls, presumably of different weight. It was explained to the participant that the actual weight differences involved were only very small so that the task was quite hard, and that they should give their best guess when undecided. During the data collection, the Experimenter sat behind a large screen. In each trial, she collected the ordered pair of balls indicated by a computer program, placed the balls on a custom-built tray, and slid the tray through an opening in the screen that was just large enough for the tray to pass through. There was no verbal or nonverbal communication (except the registered response) between the Experimenter and the participants during the entire process of data collection. The task of the participants was to lift simultaneously the balls with the thumb and index finger of the left and right hand, and to vocalize the number on the ball that appeared heavier to them. This response feature forced the participants to attend to the (otherwise task-irrelevant) numbers on the balls. The Experimenter then pulled the tray with the balls back behind the screen, and entered the verbal response into the computer. All participants compared each of the 72 ordered pairs of balls twice, for a total of 144 trials. The trial order was randomized by the computer except that there were non-overlapping blocks of 36 trials each containing all nonordered pairs exactly once.

\section{Results}

We first computed the proportion of choosing the left ball $(i)$ as heavier for each digit pair $(i, j)$ and for each participant separately. In a second step, we computed the mean response proportion for each digit pair across all 36 participants; these 72 mean response proportions are shown in Fig. 1 (top panel).

We estimated the logistic regression model for paired comparison data by maximizing the likelihood (ML) and by inverting the Fisher information matrix (for detailed background, see Agresti, 2013; Fleiss et al., 2003; Morgan, 2009: Walker et al., 2010) to derive confidence intervals (CIs). We tested our predictions in two steps: first by analyzing the mean response proportions, and then by fitting the logistic regression model to the data of each participant separately. In accordance with the findings reviewed in the Introduction, both analyses used the logged numerical distance $\ln (i)-\ln (j)=\ln (i / j)$ to predict the 72 probabilities $p_{i j}=\mathrm{P}[$ “choose left ball” $\mid(i, j)]$ for $i, j=$ $1, \ldots, 9, i \neq j$, where $i(j)$ is the number on the left (right)

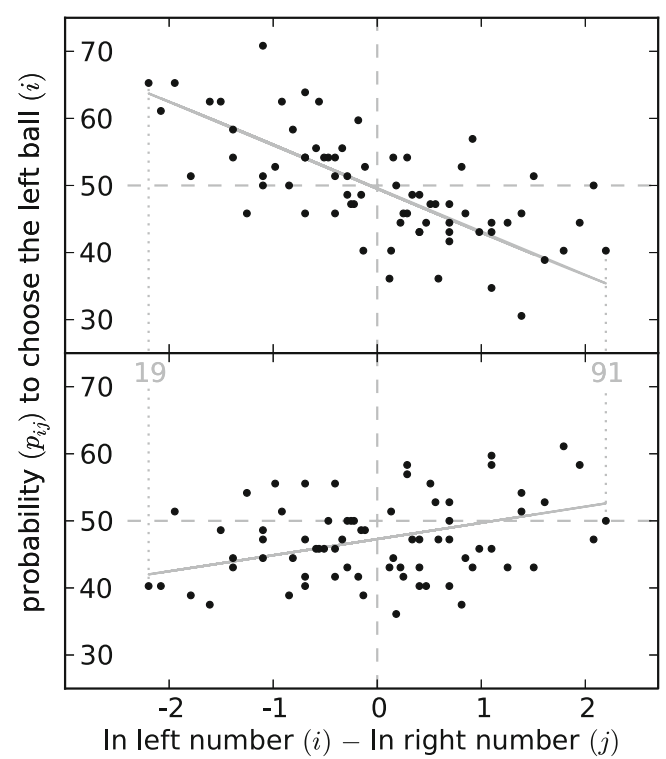

Fig. 1 The 72 mean proportions $p_{i j}$ (ordinate, in \%) of choosing the left ball, when the left ball carried number $i$ and the right ball number $j$, as a function of the (logarithmic) distance $\ln (i)-\ln (j)=\ln (i / j)$. For example, as indicated by the dotted lines, the pairs $(1,9)$ (resulting in $\ln (i / j)=-2.20$ ) and $(9,1)$ (so that $\ln (i / j)=+2.20)$ are shown as left- and rightmost data points, respectively. The solid lines show the logistic regression model. Top (bottom) panel: mean response proportions $p_{i j}$ in Experiment 1 (Experiment 2), under the instruction to choose the heavier (the lighter) ball 
ball as seen by the participant. These predictions are based on the logistic regression model

$\operatorname{logit}(i, j)=\ln \frac{p_{i j}}{1-p_{i j}}=b+k \cdot \ln (i / j)$

where $k$ is the logit slope of the response proportions with the logged numerical distance, and $b$ is a parameter indicating the overall response bias towards choosing the left ball. In particular, a finding of $k<0$ indicates that participants judged the left ball as heavier with higher probability when the number on the left ball was smaller and the number on the right ball was larger. The approach using the single-subject model fit is based on a logistic random effects model (Agresti, 2013, ch. 13; Fleiss et al., 2003, ch. 15) in which the logistic parameters $(k, b)$ for any subject are represented as a random draw from a reference population of subjects. This implies that the variance of the individual-subject estimates of, e.g., $k$ has two logically separate components: the variability of the subject-specific $k$-values, plus the (conditional) squared standard error of the estimate of $k$ per subject. Under the present design (72 pairs, 144 trials per participant), the squared standard error of the per-subject estimates is $0.17^{2}$.

The 72 averaged response proportions are shown in Fig. 1 (upper panel) as a function of the logged numerical distance $\ln (i)-\ln (j)=\ln (i / j)$. The ML estimate of the logit slope $k$ for these averaged response proportions is $k=$ -0.213 , the standard error of $k$ is 0.028 , and the $95 \%$ CI for $k$ is [ $-0.267,-0.158]$. There is thus a highly significant increase of the probability to judge the left ball as heavier as the number on the left ball gets smaller and that on the right ball larger. At the same time, no systematic response bias was observed; the ML estimate of $b=-0.010$, its standard error is 0.028 , and the $95 \% \mathrm{CI}$ for $b$ is $[-0.065,+0.045]$.

It is quite plausible that the individual participants differ by more than just binomial random error in the way in which the numbers on the balls influence their weight judgments (e.g., Miller \& Schwarz, 2018). To evaluate this aspect, we also fitted the logistic regression model to the data of each participant individually, using the logistic random effects model described above. This produced 36 independent estimates of $k$ and of $b$, a total of 72 parameters, as compared to only two parameters for the corresponding analysis based on the averaged response proportions. Comparing the maximized likelihoods ( $L_{0}$ and $L_{1}$, say) under both models, the statistic $-2 \cdot \ln \left(L_{0} / L_{1}\right)=$ 697.70, a value that would follow a $\chi^{2}$-distribution with $d f=72-2=70$ if there were no systematic betweenparticipant differences. Thus, there is highly significant evidence that participants differ in the way in which the numbers on the balls influence their judgments. To evaluate if the findings based on the averaged response proportions possibly reflect averaging artifacts, we tested the hypothesis that (across participants) the mean value of $k$ differs only by random error from zero against the alternative hypothesis that $k<0$. The mean logit slope across participants is $\bar{k}=-0.265$, its standard error is 0.064 , and the corresponding $t(d f=35)=-4.12, p<.001$. The conditional standard error of the single-subject estimates of $k$ is 0.17 , which means that only about one-half per cent of the overall variance of $\bar{k}$ is contributed by the within-subject estimation error. Compared to the analysis based on the averaged response proportions, these results indicate even more clearly that participants judged the left ball to be heavier more frequently when the number on the left ball was smaller and the number on the right ball was larger. The mean of the response bias parameter across participants is $\bar{b}=-0.019$, its standard error is 0.121 ; the corresponding $t(d f=35)=-0.154, p>.87$, indicates that on average there was no systematic bias towards choosing the left or right ball ${ }^{1}$

\section{Discussion}

The results of Experiment 1 are clearly in line with the interpretation derived in the Introduction: smaller numbers are associated with smaller perceived size of the balls, and smaller size in turn is associated with greater weight. According to the hypothesized number-weight illusion both effects combine to produce the effect that we have seen both in analyzing the averaged response proportions and very similarly in analyzing the number-weight relation in each participant individually.

Although this interpretation via a size-mediated influence of numbers on weight perception is strongly supported by the massive evidence reviewed in the Introduction, alternative interpretations remain logically tenable. For example, the numbers might influence the perception of the ball's hardness, which in turn might influence the perception of weight ${ }^{2}$. Alternatively, larger numbers might be experienced as "higher" and smaller numbers as "lower" (e.g., Walker, Scallon, \& Francis, 2017; also see Walker, Walker, \& Francis, 2012, 2015), while lighter objects tend to be higher up in the environment and heavier objects tend to be lower ${ }^{3}$. In combination, these number-height and height-weight associations would lead to a similar pattern as observed in Experiment 1. A corresponding argument might be made for the ball's perceived density (cf., Peters

\footnotetext{
${ }^{1}$ Graphs of the distribution of the single-subject estimates of $k$ and $b$ in Experiment 1 are provided in the Supplemental Materials.

${ }^{2}$ We thank an anonymous reviewer for suggesting this interpretation.

${ }^{3}$ We thank another anonymous reviewer for suggesting this interpretation.
} 
et al., 2016). Finally, digits might also directly influence the expected weight of the balls shown, with no mediation via perceived size. If higher numbers are associated with higher expected weight, this would lead to an expectation-mediated effect as in the empty suitcase illusion mentioned in the Introduction.

Logically at least, there is yet another alternative view of the results in Experiment 1, however. It is a necessary and intended feature of our design that the numbers on the balls were clearly visible; thus, one might argue that participants have a systematic response bias to name that ball in the pair which has the smaller number, quite independent of the requirements of the weight comparison task. It is not exactly apparent why this form of bias should exist, but it is a fact that by design (i.e., zero weight differences) our weight comparison task was extremely difficult, which arguably left more room for biased response strategies. Even though according to the ATOM model reviewed in the Introduction one would rather expect a bias of the opposite polarity (i.e., small numbers associated with "lighter" rather than "heavier"), it remains nevertheless true that judgments consistently generated with this form of response bias would equally produce results as in Fig. 1 (top).

To test if the results seen in Fig. 1 reflect genuine number-weight relations or simply the form of response bias just described we repeated Experiment 1 with a single change in design: the participants now had to choose the lighter of the two balls presented. If indeed participants have a systematic bias to name that ball in the pair which carries the smaller number then we would expect that the results in Experiment 2 should be quite similar to those in Experiment 1: as before, participants should preferentially choose the ball with the smaller number, independent of the requirements of the weight comparison task. On this view, the probability to choose the left ball (as being lighter) should as before decrease with the number $(i)$ on the left and increase with the number $(j)$ on the right ball, i.e., the logit slope $k$ should again tend to be negative. On the other hand, according to the hypothesized number-weight illusion we would expect that the left ball appears as lighter if the number $i$ on the left ball is large and the number $j$ on the right ball is small. That is, the probability to choose the left ball (as being lighter) should then increase with the number $(i)$ on the left, and decrease with the number $(j)$ on the right ball, i.e., the logit slope $k$ should now tend to be positive.

\section{Experiment 2}

Experiment 2 was a complete paired-comparison task in which participants judged which of two numbered balls appeared to be lighter.

\section{Method}

All aspects were exactly as in Experiment 1, except that the participants were now instructed to name the number on the ball that they judged to be lighter.

\section{Results}

The 72 averaged response proportions are shown in Fig. 1 (lower panel) as a function of the logged numerical distance $\ln (i)-\ln (j)=\ln (i / j)$. The ML estimate of the logit slope for these averaged responses proportions is $k=+0.070$, the standard error of $k$ is 0.027 , and the $95 \%$ C.I. for $k$ is $[+0.017,+0.124]$. There is thus a significant increase of the probability to judge the left ball as lighter as the number on the left ball gets larger and that on the right ball smaller. At the same time, a small but systematic bias towards choosing the right ball was observed; the ML estimate is $b=-0.113$, its standard error is 0.028 , and the $95 \%$ CI for $b$ is $[-0.167,-0.058]$.

To evaluate if the findings based on the averaged response proportions possibly reflect averaging artifacts, we again fitted the logistic regression model to the data of each participant individually; this produced 36 independent estimates of $k$ and of $b$. As in Experiment 1, the $\chi^{2}-$ statistic $-2 \cdot \ln \left(L_{0} / L_{1}\right)=917.06$ strongly suggests that participants differ systematically in their individual values of $k$ and $b$. We therefore tested the hypothesis that (across participants) the mean logit slope $\bar{k}$ differs only by random error from zero against the alternative hypothesis that $\bar{k}>0$. The mean slope across participants is $\bar{k}=+0.097$, its standard error is 0.055 , and the corresponding $t(d f=35)=+1.77, p<.043$. Thus, the participants judged the left ball more frequently to be lighter to the extent that the number on the left ball was larger than the number on the right ball. The mean of the response bias parameter across participants is $\bar{b}=-0.143$, its standard error is 0.157 ; the corresponding $t(d f=35)=$ $-0.908, p>.37$, indicates that when the logit model is fitted to each participant separately then on average there was no systematic bias towards choosing the left or right ball. $^{4}$

In a final analysis, we also compared the means of the 36 slope values from Experiments 1 and 2. The response bias account leading to Experiment 2 predicts that these means should not differ systematically on average, whereas the account in terms of the number-weight illusion predicts that the difference of the mean slope in Experiment $2\left(\bar{k}_{2}\right)$ minus the mean slope in Experiment $1\left(\bar{k}_{1}\right)$ should be positive. The mean difference is $\bar{k}_{2}-\bar{k}_{1}=0.362$, and an independent $t$

\footnotetext{
${ }^{4}$ Graphs of the distribution of the single-subject estimates of $k$ and $b$ in Experiment 2 are provided in the Supplemental Materials.
} 
test gives $t(d f=70)=3.95, p<.001$; the $95 \% \mathrm{CI}$ for the mean difference of the slopes in Experiments 1 and 2 is $[+0.182,+0.542]$.

\section{Discussion}

The results of Experiment 2 are again in line with the predictions derived in the Introduction: the left ball was chosen with higher probability as being lighter to the extent that the left ball had a larger number than the right ball. This was true when analyzing averaged response proportions, and also when using single-participant logistic analyses of the number-weight relation. Both findings correspond to the hypothesized number-weight illusion, and they are inconsistent with the alternative response bias account according to which participants have a general tendency to select the ball with the smaller number on it.

In post-experimental interviews, several participants claimed they would find it "more natural" to try to determine which ball was heavier (rather than lighter), and to some degree this attitude may be reflected in the lower absolute value of $k$ in Experiment 2 as compared to Experiment 1. The finding is reminiscent of similar instruction-related effects in weight comparisons, e.g., (Duncan \& Sheppard, 1963; Ross \& Gregory, 1964), and also to standard findings in numerical comparison studies in which "choose larger" typically produces faster RTs and more consistent effects (e.g., Banks et al., 1976,Schwarz \& Stein, 1998).

\section{General discussion}

The present study describes a new number-weight illusion that is directly interpretable as a combination of two wellestablished findings: the classical size-weight illusion, and the strong consistent association of numerical magnitude and physical size. Smaller numbers are associated with smaller size, and balls of smaller perceived size appear as heavier than otherwise identical balls which appear bigger.

As pointed out in the Discussion of Experiment 1, alternative interpretations remain logically tenable according to which the perception of other properties than size such as, e.g., the ball's hardness mediate the perception of its weight. However, as reviewed in the Introduction, the interpretation based on a size-mediated influence on weight perception seems clearly more in line with extant evidence that was obtained under conditions directly comparable to the present experiments, and with participants of similar age. A relevant characteristic comment given independently by several of our participants in post-experimental interviews was that the already hard weight comparison task was made even more difficult because, according to their impression, "the balls also differed in size". This corresponds to the often-confirmed and robust findings from numbersize congruency experiments reviewed in the Introduction demonstrating that larger (smaller) numbers are strongly associated with larger (smaller) physical size. In fact, in this broader perspective our findings connect well to classical studies (e.g., Bruner \& Goodman, 1947) demonstrating that, e.g., the perceived diameter of coins is consistently influenced by the value indicated on it, not unlike the numbered balls in the present study (for corresponding recent grip aperture studies on how numbers influence perceived object size under conditions closely approximating those of the present study, see Andres et al., 2008, and Viarouge \& de Hevia, 2013).

Ross and Gregory (1964) demonstrated that the Weber ratio in psychophysical weight judgments depends on perceived rather than on physical weight. Since then many physical variables (e.g., Buckingham et al., 2016; Walker, Francis, \& Walker, 2010) and task conditions (e.g., Masin \& Crestoni, 1988) have been identified which influence how weight is perceived. To these findings the present study adds that (in one plausible interpretation, via perceived size) apparent weight can also be systematically influenced by purely symbolic features, that is, by numbers whose abstract meaning cannot be deduced from their outer physical appearance but must be retrieved from long-term memory. Our findings thus fit in well with the view (Flanagan \& Beltzner, 2000; Dijker, 2014) that the reafference account is not limited to the sensorimotor level but operates as well at perceptual and cognitive levels (for recent examples, see, e.g., Buckingham \& MacDonald, 2016, Dijker, 2008, Zhu \& Bingham, 2011).

From the perspective of numerical cognition, our results add to the list of findings that numerical magnitude not only affects purely cognitive judgments about the abstract order relations of numbers but extends to more versatile tasks in which motor efference copies are compared to sensory feedback. Our findings thus complement and extend earlier studies demonstrating that symbolic numerical magnitude systematically influences complex activities such as visual search (Schwarz \& Eiselt, 2012; Sobel, Puri, \& Faulkenberry, 2016), the scaling of manual force in motor commands (Fischer \& Miller, 2008; Lindemann et al., 2007; Rugani et al., 2017), or the evaluation of the temporal order of events (Gevers et al., 2003; Müller \& Schwarz, 2008; Schwarz \& Eiselt, 2009). In contrast to these findings the present study is the first to report the a priori seemingly implausible finding shown in Fig. 1 that larger numerical magnitudes go with less of a perceived physical quantity (weight). This, however, corresponds precisely to what we should expect on the basis of two well-established perceptual relations: the association of number and size on the one hand, and of size and weight on the other would combine to produce the observed number-weight illusion. 
Acknowledgements We would like to thank Prof. Jeff Miller, University of Otago, New Zealand, for discussion and Madeleine Scholz for collecting the data.

The present work was supported by a research grant (SCHW 611/5-1) from the Deutsche Forschungsgemeinschaft (DFG).

\section{References}

Agresti, A. (2013). Categorical data analysis, (3rd ed.). New York: Wiley.

Andres, M., Ostry, D. J., Nicol, F., \& Paus, T. (2008). Time course of number magnitude interference during grasping. Cortex, 44 , 414-419.

Banks, W. P., Fujii, M., \& Kayra-Stuart, F. (1976). Semantic congruity effects in comparative judgments of magnitudes of digits. Journal of Experimental Psychology: Human Perception and Performance, 2, 435-447.

Besner, D., \& Coltheart, M. (1979). Ideographic and alphabetic processing in skilled reading of English. Neuropsychologia, 17, 467-472.

Bonato, M., Zorzi, M., \& Umiltà, C. (2012). When time is space: Evidence for a mental time line. Neuroscience \& Biobehavioral Reviews, 36, 2257-2273.

Bruner, J. S., \& Goodman, C. C. (1947). Value and need as organizing factors in perception. Journal of Abnormal and Social Psychology, 42, 33-44.

Buckingham, G., \& MacDonald, A. (2016). The weight of expectation: Implicit, rather than explicit, prior expectations drive the size-weight illusion. The Quarterly Journal of Experimental Psychology, 69, 1831-1841.

Buckingham, G., Goodale, M. A., White, J. A., \& Westwood, D. A. (2016). Equal-magnitude size-weight illusions experienced within and between object categories. The Journal of Vision, 16:25, 1-9.

Bueti, D., \& Walsh, V. (2009). The parietal cortex and the representation of time, space, number and other magnitudes. Philosophical Transactions of the Royal Society, B 364, 18311840.

Butterworth, B. (1999). The mathematical brain. London: Macmillan.

Cohen Kadosh, R., Cohen Kadosh, K., Linden, D. E. J., Gevers, W., Berger, A., \& Henik, A. (2007). The brain locus of interaction between number and size: A combined functional magnetic resonance imaging and event-related potential study. Journal of Cognitive Neuroscience, 19, 957-970.

Dehaene, S. (2003). The neural basis of the Weber-Fechner law: A logarithmic mental number line. Trends in Cognitive Sciences, 7 , 145-147.

Dehaene, S., \& Brannon, E. M. (2011). Eds. Space time and number in the brain: Searching for the foundations of mathematical thought. Amsterdam: Academic Press.

Dijker, A. J. M. (2008). Why Barbie feels heavier than Ken: The influence of size-based expectancies and social cues on the illusory perception of weight. Cognition, 106, 1109-1125.

Dijker, A. J. M. (2014). The role of expectancies in the size-weight illusion: a review of theoretical and empirical arguments and a new explanation. Psychonomic Bulletin and Review, 21, 1404-1414.

Duncan, C. J., \& Sheppard, P. M. (1963). Continuous and quantal theories of sensory discrimination. Proceedings of the Royal Society of London B, 158, 343-363.

Fischer, R., \& Miller, J. (2008). Does the semantic activation of quantity representations influence motor parameters?. Experimental Brain Research, 189, 379-391.

Fitousi, D. (2014). On the internal representation of numerical magnitude and physical size. Experimental Psychology, 61, 149-163.
Flanagan, J. R., \& Beltzner, M. A. (2000). Independence of perceptual and sensorimotor predictions in the size-weight illusion. Nature Neuroscience, 3, 737-741.

Fleiss, J. L., Levin, B., \& Paik, M. C. (2003). Statistical methods for rates and proportions, (3rd ed.). New York: Wiley.

Gallistel, C. R., \& Gelman, R. (2000). Nonverbal numerical cognition: from reals to integers. Trends in Cognitive Science, 4, 59-65.

Gallistel, C. R., \& Gelman, R. (2005). Mathematical cognition. In Holyoak, K., \& Morrison, R. (Eds.), Cambridge handbook of thinking and reasoning (pp 559-588). New York: Cambridge University Press.

Galton, F. (1880). Visualised numerals. Nature, 21, 252-256.

Gevers, W., \& Reynvoet, B. (2003). The mental representation of ordinal sequences is spatially organized. Cognition, 87, B87-B95.

Gregory, R. L. (2004). Ed. The Oxford companion to the mind, (2nd ed.). Oxford: Oxford University Press.

Gregory, R. L. (2015). Eye and brain: The psychology of seeing, (5th ed.). Princeton: Princeton University Press.

Helmholtz, H. (1910). Handbuch der physiologischen Optik, vol. III (3rd ed.) [Handbook of physiological optics]. Eds. Gullstrand, A, von Kries, J., and Nagel W. Hamburg and Leipzig: Voss.

Henik, A., Leibovich, T., Naparstek, S., Diesendruck, L., \& Rubinsten, O. (2012). Quantities, amounts, and the numerical core system. Frontiers in Human Neuroscience, 5, 186.

Henik, A., \& Tzelgov, J. (1982). Is three greater than five: The relation between physical and semantic size in comparison tasks. Memory \& Cognition, 10, 389-395.

Holst, E., \& Mittelstaedt, H. (1950). Das Reafferenzprinzip [The reafference principle]. Naturwissenschaften, 37, 464-476.

Lambrechts, A., Walsh, V., \& Wassenhove, V. (2013). Evidence accumulation in the magnitude system. Plos One, 8, e82122.

Lindemann, O., Abolafia, J. M., Girardi, G., \& Bekkering, H. (2007). Getting a grip on numbers: Numerical magnitude priming in object grasping. Journal of Experimental Psychology: Human Perception and Performance, 33, 1400-1409.

Maloney, E. A., Risko, E. F., Preston, F., Ansari, D., \& Fugelsang, J. (2010). Challenging the reliability and validity of cognitive measures: The case of the numerical distance effect. Acta Psychologica, 134, 154-161.

Masin, S. C., \& Crestoni, L. (1988). Experimental demonstration of the sensory basis of the size-weight illusion. Perception \& Psychophysics, 44, 309-312.

Miller, J., \& Schwarz, W. (2018). Implications of individual differences in on-average null effects. Journal of Experimental Psychology: General, 147, 377-397.

Morgan, B. J. T. (2009). Applied stochastic modelling, (2nd ed.). London: Chapman \& Hall.

Müller, D., \& Schwarz, W. (2008). "1-2-3": Is there a temporal number line? Evidence from a serial comparison task. Experimental Psychology, 55, 143-150.

Nieder, A. (2005). Counting on neurons: The neurobiology of numerical competence. Nature Reviews Neuroscience, 6, 177-190.

Peters, M. A. K., Ma, W. J., \& Shams, L. (2016). The sizeweight illusion is not anti-Bayesian after all: A unifying Bayesian account. PeerJ, 4, e2124.

Reike, D., \& Schwarz, W. (2016). One model fits all: Explaining many aspects of number comparison within a single coherent model A random walk account. Journal of Experimental Psychology: Learning, Memory and Cognition, 42, 1957-1971.

Reike, D., \& Schwarz, W. (2017). Exploring the origin of the numbersize congruency effect: Sensitivity or response bias?. Attention, Perception \& Performance, 79, 383-388.

Ross, H. E., \& Gregory, R. L. (1964). Is the Weber fraction a function of physical or perceived input?. The Quarterly Journal of Experimental Psychology, 16, 116-122. 
Rugani, R., \& de Hevia, M.-D. (2017). Number-space associations without language: Evidence from preverbal human infants and non-human animal species. Psychonomic Bulletin \& Review, 24, 352-369.

Rugani, R., Betti, S., Ceccarini, F., \& Sartori, L. (2017). Act on Numbers: Numerical magnitude influences selection and kinematics of finger movement. Frontiers in Psychology, 8, 1481.

Schwarz, W., \& Eiselt, A. K. (2009). The perception of temporal order along the mental number line. Journal of Experimental Psychology: Human Perception and Performance, 35, 989-1004.

Schwarz, W., \& Eiselt, A. K. (2012). Numerical distance effects in visual search. Attention, Perception, \& Psychophysics, 74, 1098-1103.

Schwarz, W., \& Ischebeck, A. (2003). On the relative speed account of number-size interference effects in comparative judgments of numerals. Journal of Experimental Psychology: Human Perception and Performance, 29, 507-522.

Schwarz, W., \& Stein, F. (1998). On the temporal dynamics of digit comparison. Journal of Experimental Psychology: Learning, Memory, and Cognition, 24, 1275-1293.

Sobel, K. V., Puri, A. M., \& Faulkenberry, T. J. (2016). Bottom-up and top-down attentional contributions to the size congruity effect. Attention, Perception, \& Psychophysics, 78, 1324-1336.

Viarouge, A., \& de Hevia, M. D. (2013). The role of numerical magnitude and order in the illusory perception of size and brightness. Frontiers in Psychology, 4, 484.
Walker, P., Francis, B. J., \& Walker, L. (2010). The brightness-weight illusion - darker objects look heavier but feel lighter. Experimental Psychology, 57, 462-469.

Walker, P., Walker, L., \& Francis, B. (2015). The size-brightness correspondence: Evidence for crosstalk among aligned conceptual feature dimensions. Attention, Perception \& Performance, 77, 2694-2710.

Walker, P., Walker, L., \& Francis, B. (2012). A common scheme for cross-sensory correspondences across stimulus domains. Perception, 41, 1186-1192.

Walker, P., Scallon, G., \& Francis, B. (2017). Cross-sensory correspondences: Heaviness is dark and low-pitched. Perception, 46, 772-792.

Walsh, V. (2003). A theory of magnitude: common cortical metrics of time, space and quantity. Trends in Cognitive Sciences, 7 , 483-488.

Whalen, J., Gallistel, C. R., \& Gelman, R. (1999). Nonverbal counting in humans: The psychophysics of number representation. Psychological Science, 10, 130-137.

Zhu, Q., \& Bingham, G. P. (2011). Human readiness to throw: the sizeweight illusion is not an illusion when picking the best objects to throw. Evolution and Human Behaviour, 32, 288-293. 